\title{
An Enhanced Response Time Mechanism in Grid Systems
}

\author{
SeongHoon Lee \\ Communication \& Information Division \\ Baekseok University, Cheonan City, Choongnam, Korea
}

\begin{abstract}
For applications that are grid enabled, the grid can offer a resource balancing effect by scheduling grid jobs on machines with low utilization. When jobs communicate with each other, the internet, or with storage resources, an advanced scheduler could schedule them to minimize communications traffic or minimize the distance of the communications. We propose an intelligent load distribution algorithm to minimize communications traffic and distance of the communications using genetic algorithm. The experiments show the proposed load redistribution algorithm performs efficiently in the variance of load in grid environments.
\end{abstract}

Keywords: Grid Systems, Resource Management, Scheduling, Intelligent Load Distribution.

\section{INTRODUCTION}

A grid federates a large number of resources contributed by individual machines into a large single-system image. For applications that are grid enabled, the grid can offer a resource balancing effect by scheduling grid jobs on machines with low utilization[1, 2, 3, 5]. This feature can prove invaluable for handling occasional peak loads of activity in parts of a larger organization. This can happen in the ways: An unexpected peak can be routed to relatively idle machines in the grid[2, 3, 5]. Other more subtle benefits can occur using a grid for load redistribution. When jobs communicate with each other, the internet, or with storage resources, an advanced scheduler could schedule them to minimize communications traffic or minimize the distance of the communications. This can potentially reduce communication and other forms of contention in the grid. An objective of load redistribution in grid environment is to allocate tasks among the processors to maximize the utilization of processors and to minimize the mean response time. In a dynamic scheme, an overloaded processor(sender) sends excess tasks to an underloaded processor(receiver) during execution.

Load redistribution activity is initiated by a sender trying to send a task to a receiver in sender-based systems[7, 8, 9]. Decision of task transfer is made in each processor independently. A request message for the task transfer is initially issued from a sender to another processor randomly selected. If the selected processor is receiver, it returns an accept message. And the receiver is ready to receive an additional task from the sender. Otherwise, it returns a reject message, and the sender tries for others until receiving an accept message. When the grid environment becomes heavy

\footnotetext{
${ }^{*}$ Corresponding author.E-mail : shlee@bu.ac.kr

Manuscript received May. 03, 2009 ; accepted Jun. 22, 2010
}

system load, it is difficult to find a suitable receiver because most processors have additional tasks to send. So, many request and reject messages are repeatedly sent back and forth, and a lot of time is consumed before execution.

To solve these problems in sender-based algorithm, we use a new genetic algorithm. In this scheme, a number of request messages issued before accepting a task are determined through a proposed genetic algorithm.

\section{PROPOSED ALGORITHM}

\subsection{Factors to be considered}

We employ the CPU queue length as a suitable load index because this measure is known as a most suitable index[8]. This measure means a number of tasks in CPU queue residing in a processor. We use a 3-level scheme to represent a load state on its own CPU queue length of a processor. In 3-level scheme, $\mathrm{T}_{\text {up }}$ and $\mathrm{T}_{\text {low }}$ are algorithm design parameters and are called upper and lower thresholds respectively.

Transfer policy uses the threshold policy that makes decision based on CPU queue length(CQL). The transfer policy is triggered when a task arrives. A node identifies as a sender if a new task originating at the node makes the CPU queue length exceed $\mathrm{T}_{\text {up }}$. A node identifies itself as a suitable receiver for a task acquisition if the node's CPU queue length will not cause to exceed $\mathrm{T}_{\text {low }}$.

Each processor in grid environment has its own population which genetic operators are applied to. There are many encoding methods. We use binary encoding method in this paper. So, a string in population can be defined as a binarycoded vector $\left\langle\mathrm{v}_{\mathrm{o}}, \mathrm{v}_{1}, \ldots, \mathrm{v}_{\mathrm{n}-1}\right\rangle$ which indicates a set of processors to which the request messages are sent off. If the request message is transferred to the processor $\mathrm{P}_{\mathrm{i}}($ where $0 \leq \mathrm{i} \leq \mathrm{n}-1, \mathrm{n}$ is the total number of processors), then $\mathrm{v}_{\mathrm{i}}=1$, otherwise $\mathrm{v}_{\mathrm{i}}=0$. 


\subsection{Algorithm Approach}

In the sender-based load redistribution approach using genetic algorithm, processors received the request message from the sender send accept message or reject message depending on its own CPU queue length. In the case of more than two accept messages returned, one is selected at random. Each string included in a population is evaluated by the fitness function using following formula.

$$
F_{i}=1 /\left(\left(\alpha^{*} \mathrm{TMP}\right)+\left(\beta^{*} \mathrm{TMT}\right)+\left(\gamma^{*} \mathrm{TTP}\right)\right)
$$

Here, $\alpha, \beta, \gamma$ mean the weights for parameters such as TMP, $T M T, T T P$. The purpose of the weights is to be operated equally for each parameter to fitness function $F_{i}$.

$T M P$ (Total Message Processing time) is the summation of the processing times for request messages to be transferred. TMT(Total Message Transfer time) means the summation of each message transfer times from the sender to processors corresponding to bits set ' 1 'in selected string. The objective of this parameter is to select a string with the shortest distance eventually. TTP(Total Task Processing time) is the summation of the times needed to perform a task at each processor corresponding to bits set ' 1 ' in selected string. The objective of this parameter is to select a string with the fewest loads. Eventually, a string with the largest fitness value in population is selected. And after genetic_operation is performed, the request messages are transferred to processors corresponding to bits set ' 1 ' in selected string.

The Fig. 1 shows our proposed general algorithm.

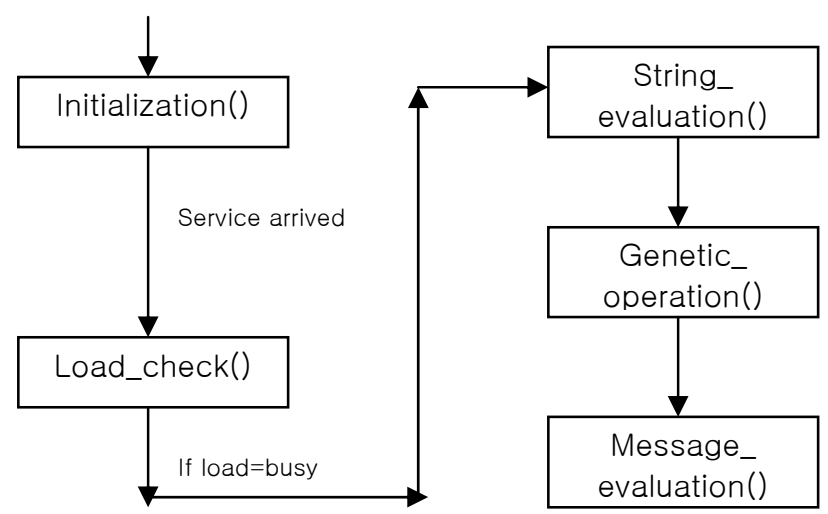

Fig. 1. General Algorithm

This algorithm consists of five modules such as Initialization, Load_check, String_evaluation, Genetic_operation and Message_evaluation. These modules are executed at each processor in grid environment. The algorithm of the proposed sender-based load redistribution is as following.

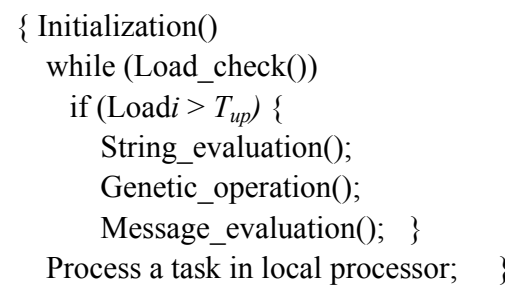

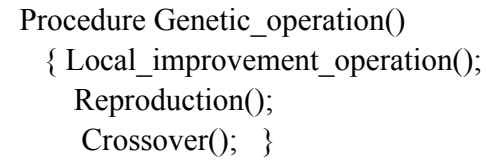

An Initialization module is executed in each processor. A population of strings is randomly generated without duplication. A Load_check module is used to observe its own processor's load by checking the CPU queue length, whenever a task is arrived in a processor. If the observed load is heavy, the load redistribution algorithm performs the above modules. A String_evaluation module calculates the fitness value of strings in the population. A Genetic_operation module such as Local improvement, Reproduction, Crossover is executed on the population in such a way as above. Grid environment consists of groups with autonomous computers. When each group consists of many processors, we can suppose that there are $p$ parts in a string corresponding to the groups. The following genetic operations are applied to each string, and new population of strings is generated:

In local improvement operation (), string 1 is chosen. A copy version of the string 1 is generated and part 1 of the newly generated string is mutated. This new string is evaluated by proposed fitness function. If the evaluated value of the new string is higher than that of the original string, replace the original string with the new string. After this, the local improvement of part 2 of string 1 is done repeatedly. This local improvement is applied to each part one by one. When the local improvement of all the parts is finished, new string 1 is generated. String 2 is then chosen, and the above-mentioned local improvement is done. This local improvement operation is applied to all the strings in population.

In reproduction(), the reproduction operation is applied to the newly generated strings. We use the "wheel of fortune" technique[6].

In crossover(), the crossover operation is applied to the newly generated strings. These newly generated strings are evaluated. One-point crossover used in this paper differs from the pure one-point crossover operator. In pure one-point crossover, crossover activity generates based on randomly selected crossover point in the string. But boundaries between $\operatorname{parts}(p)$ are used as an alternative of crossover points. So we select a boundary among many boundaries at random. And a selected boundary is used as a crossover point. Its purpose is to preserve effect of the local improvement operation of the previous phase.

Suppose that there are 5 parts in grid environment. A boundary among the many boundaries $\left(B_{1}, B_{2}, B_{3}, B_{4}\right)$ is determined at random as a crossover point. If a boundary $B_{3}$ is selected as a crossover point, crossover activity generate based on the $B_{3}$. So, the effect of the local improvement operation in the previous phase is preserved through crossover activity.

The Genetic_operation selects a string from the population at the probability proportional to its fitness, and then sends off the request messages according to the contents of the selected string.

A Message_evaluation module is used whenever a processor receives a message from other processors. When a processor $P_{i}$ receives a request message, it sends back an accept or reject message depending on its CPU queue length. 
Suppose that there are 10 processors in distributed systems, and the processor $P_{2}$ is a sender. Then, genetic algorithm is performed to decide a suitable receiver. And it is selected a string by a probability proportional to its fitness value. Suppose a selected string $\mathrm{S}_{5}$ is $\langle 1,0,-, 1,1,0,1,0,1,0>$, then the sender $P_{2}$ sends request messages to the processors $\left(P_{0}, P_{3}, P_{4}\right.$, $\left.P_{6}, P_{8}\right)$. After each processor $\left(P_{0}, P_{3}, P_{4}, P_{6}, P_{8}\right)$ receives a request message from the processor $P_{2}$, each processor checks its load state. If the processor $P_{0}$ is a light load state, the processor $P_{0}$ sends back an accept message to the processor $P_{2}$. Then the processor $P_{2}$ transfers a task to the processor $P_{0}$.

\begin{tabular}{|c|c|c|}
\hline \multicolumn{3}{|c|}{$\begin{array}{c}\text { Applied Genetic_operation( ) } \\
\downarrow \\
\text { (Example for processor } P_{2} \text { ) }\end{array}$} \\
\hline $\begin{array}{c}\text { String } \\
\text { No }\end{array}$ & String content & $\begin{array}{c}\text { Fitness } \\
\text { value }\end{array}$ \\
\hline$S_{1}$ & $0,0,-, 0,1,0,0,0,0,0$ & 0.1 \\
\hline$S_{2}$ & $1,0,-, 0,0,0,0,0,0,1$ & 0.2 \\
\hline$S_{3}$ & $0,0,-, 1,0,1,1,0,0,1$ & 0.5 \\
\hline$S_{4}$ & $0,0,-, 0,1,0,0,1,0,1$ & 0.3 \\
\hline$S_{5}$ & $1,0,-, 1,1,0,1,0,1,0$ & 0.9 \\
\hline$S_{6}$ & $1,0,-, 0,0,1,0,1,0,0$ & 0.6 \\
\hline$S_{7}$ & $0,1,-, 1,1,0,0,0,0,1$ & 0.4 \\
\hline$S_{8}$ & $0,1,-, 0,1,0,1,0,0,1$ & 0.6 \\
\hline$S_{9}$ & $1,0,-, 0,1,0,0,1,1,0$ & 0.8 \\
\hline & $\begin{array}{c}\qquad \\
\text { String selection } \\
\downarrow \\
\text { ect } S_{5} \text { (fitness value highe } \\
\downarrow \\
\text { est service to } \mathrm{P}_{0}, \mathrm{P}_{3}, \mathrm{P}_{4}, \mathrm{P}\end{array}$ & \\
\hline
\end{tabular}

Fig. 2. Algorithm processing flow

\section{EXPERIMENTS AND ANALYSIS}

Our experiments have the following assumptions. First, each task size and task type are the same. Second, the number of $\operatorname{parts}(p)$ in a string is five. The values of these parameters $P_{c}$, $P_{m}$ were known as the most suitable values in various applications[6]. The table 1 shows the detailed contents of parameters used in our experiments. The load rating over the systems is about 60 percent. The number of tasks to be performed is 3000 .

Table 1. Experimental parameters

\begin{tabular}{|l|c|}
\hline Number of processor & 24 \\
\hline Pc & 0.7 \\
\hline Pm & 0.1 \\
\hline
\end{tabular}

\begin{tabular}{|l|c|}
\hline Number of strings & 60 \\
\hline Weight of TMP & 0.02 \\
\hline Weight of TMT & 0.01 \\
\hline Weight of TTP & 0.02 \\
\hline
\end{tabular}

[Experiment 1] We compared the performance of proposed method with a conventional method in this experiment by using the parameters on the table 1 . The experiment is to observe the change of response time..

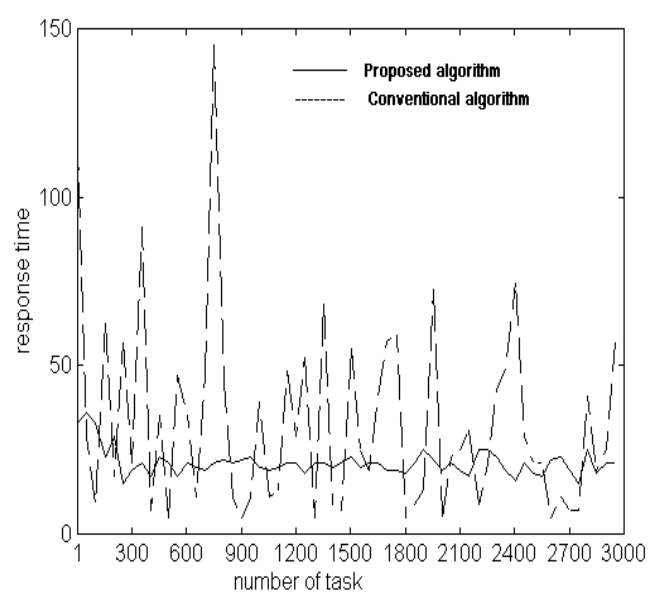

Fig. 3. Result of response time

Fig 3 shows result of the experiment 1 . In conventional method, when the sender determines a suitable receiver, it selects a processor in grid environment randomly, and receives the load state information from the selected processor. The algorithm determines the selected processor as receiver if the load of randomly selected processor is $T_{l o}$. These processes are repeated until a suitable receiver is searched. So, the result of response time shows the severe fluctuation. In the proposed algorithm, the algorithm shows the low response time because the load redistribution activity performs the genetic_operation considering the load state when it determines a receiver.

[Experiment 2] These experiments are to observe the performance when the probability of crossover is changed and are to observe the performance when the probability of mutation is changed.

Fig 4 shows the result of response time depending on the changes of $P_{c}$ when $P_{m}$ is 0.05 . It shows high performance respectively when $P_{c}$ is 0.7 . Fig 5 shows the result of the response time depending on the changes of $P_{m}$ when $\mathrm{P}_{\mathrm{c}}$ is 0.7 . The result shows high performance respectively when $P_{m}$ is 0.1 . 


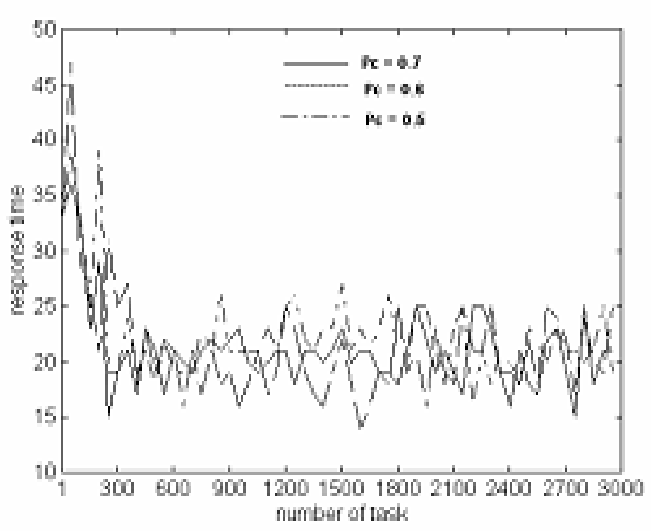

Fig. 4. Result depending on the changes of $P_{c}$

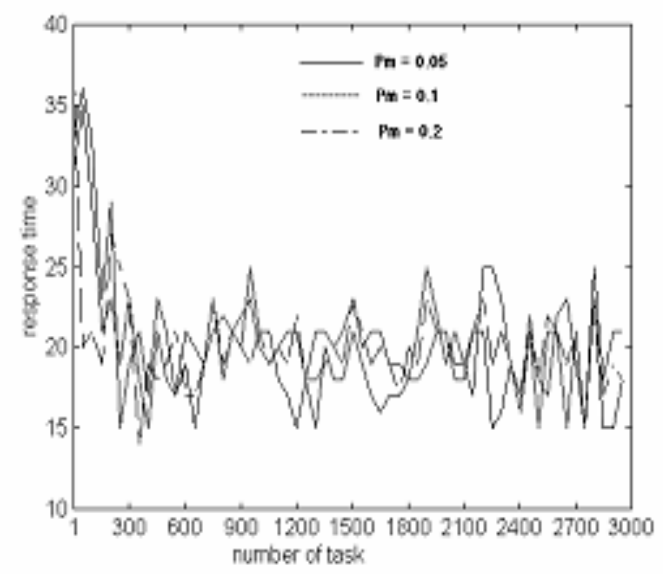

Fig. 5. Result depending on the changes of $P_{m}$

Through these several experiments, we verify that the performance of the proposed scheme is better than that of the conventional scheme. The performance depending on the changes for parameters $P_{c}$ and $P_{m}$ is excellent than that of the conventional method.

\section{CONCLUSIONS}

We proposed a new intelligent load redistribution scheme in distributed system that is based on a genetic algorithm. Several experiments have been done to compare the proposed scheme with a conventional algorithm. The various experiments proved that performances of the proposed scheme are better than those of the conventional scheme on in response time and mean response time. However the proposed algorithm is sensitive to the weight values of TMP, TMT and TTP. For a further research, a study on the method for releasing the sensitivity of weight values is suggested.

\section{REFERENCES}

[1] B. Jacob, M. Brown., Introduction to grid computing. ibm.com/redbooks.

[2] B. Jacob, M. Brown, K. Fukui, "Introduction to Grid
Computing," IBM Redbooks, http://ibm.com/redbooks.

[3] Y, Li, Z. Lan, "A Survey of Load Balancing in Grid Computing," LNCS 3314, Dec 2004, pp. 280-285

[4] http://www.gridforum.org.

[5] J. Joseph, C. Fellenstein., Grid Computing, IBM Press.

[6] J.Grefenstette, "Optimization of Control Parameters for Genetic Algorithms," IEEE Trans on SMC, vol. SMC-16, no.1, January 1986, pp.122-128.

[7] D.L.Eager, E.D.Lazowska, J.Zahorjan, "Adaptive Load Sharing in Homogeneous Distributed Systems," IEEE Trans on Software Engineering, vol.12, no.5, May 1986, pp.662-675.

[8] N. G.Shivaratri, P.Krueger, and M.Singhal, "Load Distributing for Locally Distributed Systems," IEEE COMPUTER, vol.25, no.12, December 1992, pp.33-44.

[9] T. Kunz, "The Influence of Different Workload Descriptions on a Heuristic Load Balancing Scheme," IEEE Trans on Software Engineering, vol.17, No.7, July 1991, pp.725-730.

[10] Thomas G. R., Ten Reasons to Use Divisible Load Theory. IEEE Computer Vol. 36, No. 5, 2003, pp. 63-68.

[11] http://www.cs.wisc.edu/condor.

[12] http://www.gridforumkorea.org.

[13] http://www.globus.org/.

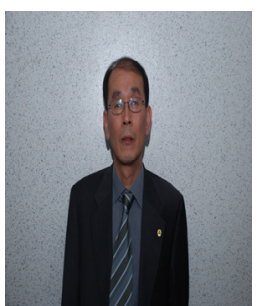

\section{SeongHoon Lee}

I received the Ph.D. in computer science from Korea University, KOREA in 1998. Since then, I has been with Baekseok Univ. Main research interests include Distributed system, Grid Computing, Web Service, etc 